\title{
L'immigration dans la littérature jeunesse Vers une compréhension du monde dans lequel on vit
}

\author{
Fanny Mahy \\ Université de Porto
}

Car dans l'immigration, c'est l'homme, la femme, les enfants qui sont en jeu. Une part d'humanité commune, universelle, qui peut et doit être évoquée et dite à tout âge, comme une compréhension du monde dans lequel on vit. Benoît Falaise, postface de l'ouvrage Vivons ensemble. Pour répondre aux questions des enfants sur l'immigration

Partageant ce constat de la possibilité, ou plutôt du devoir d'évocation, voire d'exploration de l'immigration à tout âge, nous projetons de sonder un corpus de six ouvrages de 
littérature destinés à la jeunesse dont la thématique centrale est celle de l'immigration : Akim court (2012) de Claude K. Dubois, La fille qui parle à la mer / Le garçon au chien parlant (2013) de Claudine Galea, Les deux vies de Ning (2013) et João ou l'année des révolutions (2010) de Valentine Goby, Les Vitalabri, de JeanClaude Grumberg (2014) et Le Jeu des sept cailloux (2010) de Dominique Sampiero.

Il s'agira de comprendre dans quelle mesure et comment ces livres, publiés entre 2010 et 2014, participent effectivement - ou pas - de la compréhension des enfants du monde dans lequel on vit actuellement. Les six textes retenus ont été sélectionnés sur le critère commun de la contemporanéité, mais aussi sur celui de la diversité, afin de constituer un échantillonnage représentatif des différentes pratiques observables dans la littérature migrante pour la jeunesse. Ainsi, certains d'entre eux font partie de collections spécialisées dans la thématique de l'immigration, en l'occurrence la série «Français d'ailleurs» pour Les deux vies de Ning et João ou l'année des révolutions. Les autres appartiennent à des maisons d'édition et à des collections plus éclectiques. De même, les deux titres que nous venons de mentionner s'adressent à des enfants d'" entre 9 et 13 ans » alors que d'autres textes, sans mention d'âge, semblent accessibles dès 6 ou 7 ans, et même avant, si nous prenons l'exemple de Akim court. Avec cet album aux images prédominantes, les parents pourraient lire l'histoire aux enfants tandis que ces derniers regarderaient les illustrations. On pourrait également noter la diversité géographique des personnages migrants évoluant au sein du corpus : Afrique, Chine, Portugal, Tchétchénie, aussi bien que celle des terres d'élection, qui s'étendent de l'Europe jusqu'en Amérique, même si la France reste largement prédominante. 
Nous postulons ici que ces albums, dans toute leur diversité, visent une compréhension empathique du phénomène migratoire, dans un dialogue plus ou moins prononcé entre le désir d'informer et d'émouvoir l'enfant au moyen de divers procédés esthétiques mis en œuvre, aussi bien visuels qu'écrits. Outre l'attention portée au démêlé et à la mise au jour de ces procédés, nous aurons recours à des ouvrages documentaires adressés aux enfants (à l'instar de celui susmentionné en exergue de l'article), lesquels nous permettront d'examiner si les différentes entrées thématiques qui y sont répertoriées apparaissent aussi dans la littérature jeunesse ou si certaines sont privilégiées par rapport à d'autres, mineures, voire absentes. Enfin, il s'agira surtout de vérifier si la question actuelle des difficultés du «vivre ensemble» est posée par les différents auteurs, et si oui, comment? Si non, pourquoi et quelle(s) conclusion(s) en tirer quant à la transmission de la compréhension d'un phénomène régissant le monde passé aussi bien que présent?

\section{À la découverte de la migration, comprendre les informations}

Lorsque l'enfant lit les histoires singulières de l'immigration, il n'a pas seulement accès à la particularité mais aussi à la généralité, à l'universalité à laquelle elles renvoient. Cette valeur d'exemplarité est parfois explicitement mentionnée dans les éléments paratextuels de l'album, à l'instar de Akim court, un enfant dans la guerre et Le Jeu des sept cailloux. En effet, le premier est siglé «Amnesty International » et la quatrième de couverture présente conjointement le livre et l'association : 
L'histoire d'Akim est singulière et intime. Mais elle est aussi celle de milliers d'autres enfants, hommes et femmes que la violence contraint à la fuite. Tous ont droit à la protection garantie par le droit d'asile et besoin d'elle et Amnesty International se bat pour que ce droit soit effectivement respecté partout dans le monde. (Dubois, 2012, quatrième de couverture)

De même, dans Le Jeu des sept cailloux, une postface indique: "Ceci n'est pas une fiction». S'ensuit le résumé de l'histoire d'un enfant tchétchène et de sa maman, et l'indication, dans le pénultième paragraphe, de ce que «l'histoire de Larissa est malheureusement loin d'être isolée. C'est pourquoi l'action de [leur] Collectif, créé en 1994, se cantonne depuis quelques années, par obligation, à l'aide apportée aux "sans-papiers" ». Le bas de la page mentionne les coordonnées du Collectif solidarité antiraciste et pour l'égalité des droits de SaintÉtienne-du-Rouvray.

Dans d'autres productions, la mention explicite d'exemplarité ne réside pas dans les marges paratextuelles mais dans les textes eux-mêmes, comme c'est le cas dans La fille qui parle à la mer / Le garçon au chien parlant. Cette fille, prénommée Oyana, attend un petit bateau, «enfin, pas trop petit quand même. Parce qu'ils sont nombreux à partir. La moitié de son village. La moitié de son village est descendu jusqu'à la mer.» (p. 7). Oyana est donc représentative d'un phénomène de masse ici mis en valeur par la répétition de « la moitié de son village». Si le personnage d'Oyana est effectivement investi de la charge de représenter la moitié d'un village d'Afrique, l'album Les Vitalabri étend, quant à lui, l'exemplarité d'un territoire donné à celle, illimitée, de l'ensemble des populations migrantes. La stratégie adoptée est alors celle du flou et de la relativité spatiotemporelle. Ainsi, dès 
la dixième page, le lecteur entre dans l'action qu'il lui faudra situer « un jour, dans un pays lointain ou proche, tout dépend de là où [il est lui]-même ».

Enfin, pour en revenir à Akim court, outre la mention explicite d'exemplarité relevant du paratextuel, il nous semble que la première page du texte joue sur une potentialité d'exemplarité fonctionnant par le biais du processus d'identification du jeune lecteur au jeune personnage. Ainsi, les quatre phrases de la première page pourraient se diviser en deux parties. L'initiale correspond à la description du monde d'Akim et du jeune lecteur que nous plaçons dans un pays en paix : «Dans le village d'Akim, la guerre semble loin. Akim joue tranquillement avec d'autres enfants et leurs petits bateaux au bord de la rivière Kuma ». La seconde phase, en revanche, va entraîner la différence entre la situation d'Akim et celle du jeune lecteur : «En fin d'après-midi, un bruit sourd et des tirs résonnent dans le ciel. Les grondements deviennent puissants. » Étant donné la vitesse fulgurante du passage de l'état de paix à celui de guerre, d'une phrase à la suivante, d'un moment de la journée à un autre, le jeune lecteur est amené à s'identifier au personnage avec lequel il partage la situation initiale de paix et, par le processus de projection-identification, celle de la guerre, que ne traverse pas seulement Akim mais tous les enfants vivant dans un pays militarisé. Ainsi, lorsque l'enfant lit les histoires singulières de l'immigration, il n'est pas seulement amené - au moyen de mentions explicites intra et extra-textuelles, précises ou volontairement relatives et floues - à saisir les informations relatives à un cas particulier qui serait alors cantonné à l'unicité mais à les comprendre et à les percevoir dans le prisme d'une exemplarité multipliable à souhait, ce qui étend considérablement leur portée. 
Dans certaines de ces productions à destination de la jeunesse, la compréhension du processus migratoire passe par le changement et la variété des perspectives. L'histoire s'anime de différents points de vue participant de la construction identitaire des personnages. Dans Le Jeu des sept cailloux, le personnage principal, Larissa, a quitté la Tchétchénie pour la ville de Rouen, en France. Le narrateur conte son histoire à la troisième personne en alternance avec des passages à la première personne: «Le matin, elle conduit ses enfants à l'école» et à la page suivante: "Le reste de la journée, je marche, je marche, à la recherche d'un endroit où manger, où dormir. Je marche de bureau en bureau pour remplir des papiers. Je marche pour oublier. » Les passages à la troisième personne content l'histoire de la migration de Larissa avec la distance de l'intermédiaire du narrateur tandis que ceux de la première personne nous la rendent plus proche, en tant qu'immigrée mais aussi maman, car c'est à « son ventre à peine plus gros qu'un melon, à la petite étoile qui va naître, [qu']elle raconte sa vie Là-bas, en Tchétchénie ». Ce va-et-vient entre distance et proximité invite l'enfant à l'exercice du passage du « je » $\mathrm{au}$ « il » et du « il » au « je » et donc à l'investissement de davantage de soi en l'autre et de l'autre en soi.

On retrouve ce processus dans La fille qui parle à la mer / Le garçon au chien parlant au travers de la particularité de la collection "Boomerang ». Le livre peut être lu en commençant par l'histoire La fille qui parle à la mer puis en lisant Le garçon au chien parlant ou inversement. Il suffit de retourner le livre pour s'apercevoir qu'il est impossible de déterminer laquelle des deux histoires précède l'autre. La collection joue le jeu jusqu'au bout, car les éléments paratextuels sont dédoublés : code-barre, prix, indication d'édition, etc. Ce sera donc au 
lecteur de choisir par quelle histoire il commencera le récit et lorsqu'il arrivera au milieu de l'ouvrage, il le retournera pour en lire la suite. Outre l'aspect original et ludique du procédé, le livre se voit ainsi marqué de deux perspectives : d'un côté, celle de Oyana, qui quitte son pays pour finir échouée sur une plage inconnue; de l'autre, celle d'un jeune garçon qui trouve une jolie petite fille sur la plage où il a pour habitude d'emmener son chien. Si l'enfant lecteur choisit de commencer par l'histoire de La fille qui parle à la mer, il suivra l'ordre chronologique et sera probablement captivé par l'effet vif de l'action. En revanche, avec Le garçon au chien parlant en première lecture, la migration de Oyana fera figure d'analepse et tendra donc à susciter le désir de savoir ce qui est arrivé à la jeune fille. Ainsi, chacune des deux alternatives recèle sa séduction et quoi que décide l'enfant-lecteur, nous avons donc, d'un côté, l'histoire d'une émigration, de l'autre, celle d'une immigration, telles deux facettes d'une identité en construction que le lecteur devra relier et peut-être faire tourner et retourner s'il allait jusqu'à se demander quel effet cela produirait de lire en premier le texte qu'il avait lu en second et de lire en second le texte qu'il avait lu en premier... Faire tourner et retourner, ainsi, sa compréhension du phénomène de migration.

Cette compréhension est aussi favorisée par l'enchevêtrement plus ou moins marqué entre petite et grande histoire. Ce sont dans les deux textes appartenant à la collection «Français d'ailleurs » que les références à la grande histoire sont les plus fréquentes. On s'attend à cette place de choix quand on sait que cette collection a vu le jour dans une collaboration avec la Cité nationale de l'histoire de l'immigration. En outre, le souci didactique des éditions Autrement Jeunesse les a conduites à expliciter leur collection, 
en répondant aux questions « Pourquoi? » et «Comment? ». On apprend alors notamment ceci :

Chaque titre aborde une période précise de l'histoire de France vue à travers l'histoire d'un enfant et de sa famille. Une histoire peuplée de souvenirs et d'anecdotes (le pays d'origine, le voyage, la France qu'ils découvrent), mais également marquée par les grands événements historiques de l'époque. Le récit, sous forme de fiction, mêle ainsi la petite et la grande histoire, et permet d'aborder ces thèmes sensibles par le biais de l'évocation et de l'identification. (avant-propos des ouvrages de la collection)

On trouvera un exemple de cet entrelacement entre petite et grande histoire dans Joao ou l'année des révolutions. Joao et sa famille ont fui la dictature de Salazar qui sévissait alors au Portugal mais le régime autoritaire ne se laisse pas oublier et s'immisce au cœur des pensées et des rêves de l'immigrant :

De Manuel, j'ai appris beaucoup, notamment à embrasser une fille. Son père aussi m'a appris des choses. Par exemple que Salazar et ses amis du gouvernement, au Portugal, étaient des types vraiment terribles. Qu'ils avaient envoyé une police spéciale, la PIDE, jusqu'en France, jusqu'à Champigny, et ici même peut-être, dans l'immeuble, pour espionner les émigrés portugais. Pour dénoncer les opposants comme lui, et surtout les communistes, puis pour les emprisonner si jamais ils osaient rentrer un jour au pays. Le père de Manuel, avec son corps énorme et sa voix de caverne, me glaçait le sang : la figure de Salazar est souvent revenue dans mes cauchemars. Ça y est, la lune. Faut que je rentre. Je saute sur mes pieds, je serre la main de Manuel et je pars en courant. (p. 21)

On voit dans ce passage comment la grande histoire s'intercale dans le cours de la petite et comment chacune des deux est nécessaire à la compréhension du phénomène de la migration, à la fois histoire individuelle et collective. 
Les modalités d'intégration de la grande histoire ne sont pas toutes à l'image des albums de la collection «Français d'ailleurs ». Dans celui des Vitalabri, lequel, nous l'avons vu, se déroule "un jour, dans un pays proche ou lointain », le flou spatiotemporel ne semble pas se prêter à l'intégration de la grande histoire, et pourtant, celle-ci est bien présente, en filigrane, et notamment quand l'aîné de la famille explique que le gouvernement

concocta puis édicta une loi destinée aux Vitalabri [...]. Tous et toutes devaient, sous peine de poursuites, accompagnés de leur progéniture, trop nombreuse ou pas assez, devaient se déclarer, spontanément et de leur plein gré, aux uniformes dûment assermentés formés pour, afin de se faire tatouer à la base du front, au sommet du nez, là, entre les sourcils, un V bien visible. (p. 66)

Ce passage, ancré de plein pied dans la fiction, ne peut manquer, toutefois, de renvoyer à la réalité du port de l'étoile imposé au peuple Juif durant la Seconde Guerre mondiale. Cette allusion, sur le ton de l'innocence et de la badinerie caractérisant l'ensemble de la narration, laisse entrevoir dans un contraste frappant le risque couru par les populations migrantes dès lors qu'elles ne sont plus considérées comme des êtres humains à part entière mais étiquetées et réduites à leur seul statut d'immigrants. Ainsi, par le biais de références historiques directement calquées sur la réalité ou alors fictives, mais renvoyant implicitement à une époque donnée de l'histoire, les auteurs d'albums fictionnalisant la migration privilégient l'histoire singulière mais ne la donnent à lire et à comprendre que dans un entrelacement plus ou moins marqué, mais toujours nécessaire, avec la grande histoire. 
Cette compréhension de l'histoire singulière et collective de la migration se fonde aussi sur la fonction informative des textes, laquelle repose sur divers procédés tels que les questions directes des enfants-personnages. Dans Le garçon au chien parlant, le petit Loïc voudrait comprendre l'histoire de Oyana, qu'il a trouvée échouée sur la plage. Ses parents expliquent :

Eh bien, elle arrive de très loin sans doute.

Mais d'où? a insisté Loïc.

Il y a des bateaux en ce moment qui amènent des réfugiés depuis l'Afrique.

C'est quoi, des réfugiés?

Des gens qui n'ont plus de terre ni de maison. Tu sais, dans certains pays, il y a la guerre, plus rien à manger, les gens s'enfuient, montent sur des bateaux et traversent l'océan. (p. 21-22)

La plupart des albums de notre corpus donnent à lire des histoires d'enfants-personnages, ou alors de familles, ce qui, d'une part, favorise l'identification de l'enfant-lecteur à l'enfantpersonnage et, d'autre part, facilite la mise en questions que des enfants sont toujours prompts à poser. Les questions de l'enfant-personnage deviennent ainsi les questions de l'enfantlecteur et la réponse des parents-personnages, celle que pourraient lui fournir ses propres parents. Outre les questions, la fonction informative passe aussi par des affirmations, égrenées au fil du texte, et naturellement intégrées dans la narration. Dans Les deux vies de Ning, l'enfant pourra prendre connaissance de certaines traditions et coutumes chinoises différentes de celles qu'il expérimente en France. Il pourra par exemple apprendre qu'en Chine, on change de nom quand on change de région (p. 21) ou encore que Le Parti choisit des hommes et des femmes pour les marier, ce qui fut le cas des 
parents de Ning (p. 33). Enfin, c'est dans ce livre, mais aussi dans Joao ou l'année des révolutions, que la dimension informative est la plus forte, la collection «Français d'ailleurs » fournissant systématiquement un cahier documentaire sur la période intégré à la fin de l'ouvrage. Ainsi, dans le cas des Deux vies de Ning, le dossier intitulé "l'immigration chinoise en France » comporte « des repères historiques et culturels, et des photographies d'époque, pour mieux comprendre l'histoire de Ning et celle des immigrés chinois en France» (p.69). Le dossier se compose de dix entrées portant sur la longue histoire de la migration des Chinois en France, les différentes communautés, la Chine en tant que grande puissance, la Chine du Nord-Est, l'enfant en Chine, le mineur isolé étranger, le sanspapiers, la carte de l'immigration chinoise en France, pour finir par une chronologie et un lexique. En outre, la collection «Français d'ailleurs » garantit la fiabilité de ses informations en précisant que «chaque ouvrage est relu par un historien spécialiste de la période» (avant-propos). Ainsi, par l'usage habile de questions, par des informations intelligemment dispersées, mais aussi par l'insertion de dossiers informatifs clairs et agréablement illustrés, les enfants-lecteurs d'albums de la migration sont nécessairement conduits à accroître leur curiosité et leur compréhension des mouvements de la population.

\section{Vers une compréhension des émotions, ressentir les vécus de la migration}

Comprendre la migration requiert de la part de l'enfant la mobilisation de ses facultés intellectuelles aussi bien que l'ouverture à ses émotions, avec une capacité d'identification 
favorisant le processus d'empathie. Pour l'y aider, les écrivains ont recours à divers procédés, dont les plus marquants sont l'héroïsation de personnages soumis à des conditions difficiles et aussi l'accès à leur intériorité, faite de peur, de courage, de souvenirs et de sentiments. Dans La fille qui parle à la mer, Oyana est soumise à un voyage périlleux; tous sont serrés les uns contre les autres; s'ajoutent le bruit du moteur, le froid, l'humidité, le manque de place, la pénurie de nourriture, les cris, les pleurs de ceux qui veulent rentrer chez eux, la fatigue, mais « Oyana parle dans sa tête, elle dit, Je m'en fiche d'être mouillée, j'aime bien être au bord. Je n'ai pas peur et je n'ai pas mal au cœur. » (p. 24) et le passeur rit et s'exclame devant un tel courage. Pourtant, Oyana parle à la mer de sa mère et de tous les souvenirs heureux qu'elle fait rouler dans sa tête :

Maman m'enroulait dans une couverture et m'emmenait voir les étoiles.

Maman me chantait la chanson du vent quand il passait pardessus les montagnes et balayait la terre.

Maman préparait les galettes dans la poêle avec le miel et on les mangeait tous ensemble assis sur le tapis épais.

Maman me regardait monter Noir, mon cheval, et elle applaudissait.

Maman marchait, mon petit frère dormait sur son dos, et moi je portais nos sacs.

On a marché six jours pour arriver au bord de la mer. (p. 27-28)

L'anaphore "maman» rythme le ressac des doux souvenirs d'Oyana qui viennent se briser sur la réalité d'un naufrage qui se dit sans remous, sans drame, dans la continuité anaphorique de ce qui se passe en Oyana : «Je crois que je me suis endormie. Je crois que je suis fatiguée. Je crois que la mer est folle. Je crois que je retourne au village. Je crois que je grandis d'un coup. Je crois que le bateau se renverse. Je crois que la mer me prend dans ses bras. Je crois que je nage. Je crois 
que je rêve. » (p. 28). Le lendemain, samedi, Oyana est échouée sur une plage, " et elle se dit, J'ai perdu mes chaussures. J'ai perdu le bateau, j'ai perdu les autres » mais au bas de la p(l)age, déjà, elle « entend sa propre voix murmurer, Tu n'es pas perdue Oyana, tu es de l'autre côté de la mer, regarde comme c'est beau. » (p. 31) L'anaphore tripartite « j'ai perdu » se mue ainsi en «tu n'es pas perdue » et le verbe avoir, transitif, en verbe être, intransitif. Oyana parvient à dépasser la perte en rassemblant ses forces vers l'essentiel, son être, et la vie qu'elle porte en elle.

Anne Scheider, dans son ouvrage portant sur la littérature jeunesse migrante, de l'Algérie à la France, parle ainsi de « visée testimoniale » $(2013$, p. 24$)$ et se demande si une fois celle-ci épuisée, ce type de littérature serait condamné à disparaître. Sans doute pas, si le désir de la comprendre persiste au fil des générations. Outre le courage des héros de la migration soumis à des épreuves comme celle de la perte de leur famille, ces textes témoignent aussi du sentiment de déchirement et d'entre-deux auquel se confrontent de nombreux migrants, parmi lesquels Ning. Le titre, Les deux vies de Ning, exprime d'emblée la dualité d'une vie séparée en deux, celle de Ning, un enfant chinois, et celle de Jiang, ce même enfant chinois contraint, sous peine d'expulsion et de renvoi au pays, à cacher son identité et l'existence de sa mère qui vit sans papiers en France. Ning intègre un foyer d'accueil et témoigne de ses difficultés identitaires: "Ici, je m’entraîne à être coupé en deux: Ning au-dedans, Jiang au-dehors. Ning a un père en Chine, une mère en France; Jiang est orphelin. » (p.17) Ning souffre de ne pouvoir rencontrer sa mère qu'à intervalles irréguliers, lors de brefs rendez-vous impromptus et c'est dans cette souffrance que la dichotomie identitaire entre Ning et 
Jiang ainsi que le désir de les fusionner s'exprime avec le plus de force : «- Jiang, il faut attendre. J'ai un vrai travail, mais toujours pas de papiers. Quand je rentre au travail ce soir-là, je suis jaloux de Ning. Ning avait une mère. Elle était loin mais c'était une mère. Mama, reprends-moi. Je t'en supplie reprendsmoi, Jiang, Ning, Jianing, avec toi » (p.62). Ainsi, les personnages d'enfants-immigrés, parce qu'ils sont courageux dans l'adversité, méritent et suscitent l'empathie et l'admiration des enfants-lecteurs. Ces derniers sont alors davantage enclins à s'ouvrir à l'aventure migratoire, non plus seulement d'un point de vue factuel mais humain, avec sensations, émotions, et force sentiments reliés aux thématiques de l'identité et de l'altérité.

Cette compréhension empathique repose aussi sur des oppositions marquées entre, d'une part, des données sociopolitiques, et nous pensons surtout à la problématique de la frontière, et d'autre part, des notations plus naturelles, plus humaines, plus profondes, plus vivantes, et qui s'expriment le plus souvent avec poésie dans le topos de l'union de l'homme avec le cosmos. Dans Le Jeu des sept cailloux, "Larissa vivait dans un village au bord de la frontière russe avec des fleurs et des snipers dans les arbres." Lorsque le mot "frontière » apparaît, la violence n'est jamais éloignée et la mention d'arbres avec des fleurs et des snipers feint de rassembler pour finalement mieux opposer. La rime en « eur » et la conjonction de coordination « et » accentuent la mise sur le même plan des deux termes pour en fait mieux révéler l'incongruité et la dimension antithétique des fleurs, comme élément harmonieux et naturel, et des snipers, associés au chaos, à la violence, à la destruction, au sang et à la peur. 
L'opposition se limitait ici au cadre environnemental mais dans La fille qui parle à la mer, c'est le personnage même d'Oyana qui est décrit comme étant très proche de la nature :

Elle connaît les montagnes, la neige, les loups, les aigles. Elle sait attraper les poissons dans la rivière, traire leur chèvre, trouver les œufs des poules [...] Elle devine qu'il va pleuvoir quand le ciel devient violet. Elle imite à la perfection le chant de l'alouette, le cri de la corneille et le canard siffleur. (p. 8)

Les connaissances d'Oyana témoignent d'une fusion de son être avec la nature, en opposition avec la notion, socialement et arbitrairement fabriquée, de frontière. Oyana rêve de liberté, de chevauchées, d'union entre terre et mer, et quand elle parle à la mer, elle dit: «Je galopais, la montagne rejoignait le ciel. Il n'y avait pas de bout, pas de limite. C'était beau. C'était comme toi.» (p. 12) Oyana ira jusqu'à abolir les frontières au sein même du cosmos lorsqu'elle se trouve au milieu de la mer et dit : « Il est grand le ciel. Il est comme la mer. Ils se touchent. Est-ce que la mer devient le ciel? Et le ciel la mer? » (p. 23)

Cette union du personnage émigrant avec la nature en opposition aux rigidités des frontières se retrouve aussi dans l'album Les Vitalabri. L'aîné de la famille joue de la musique avec beaucoup de talent, si bien qu'il parvient à endormir « les uniformes » et à passer la frontière. Aussitôt, « le soleil du haut $\mathrm{du}$ ciel lui fait fête, les oiseaux sifflent, cuicuitent et trillent comme pour saluer son passage. Les fruits, du haut des branches, mûrissent et se penchent vers lui, offerts. [...] et sa chanson remplit le paysage. »(p.46) Ainsi, les formalités, les restrictions, les interdictions et les expulsions auxquelles donnent lieu les frontières délimitées et gardées par les « uniformes» contrastent ici avec une nature sensible à la 
générosité musicale du petit Vitalabri, à qui elle prodigue tous ses bienfaits de fruits, soleil, cui-cui et autres festivités. Ces contrastes favorisent, à notre avis, la compréhension sensible chez l'enfant de la problématique de délimitation du territoire, avec ses œillères, ses barrières et ses frontières arbitraires.

Dans un ouvrage intitulé Guide du mieux-vivre ensemble, paru au début de 2016, Patrick Banon explique :

La globalisation nous conduit vers une forme de civilisation mondiale. Un nouvel espace où cohabitent aspirations universelles et particularismes culturels. Chacun peut y revendiquer à la fois une citoyenneté planétaire, l'appartenance à une tradition et un attachement local. Cette situation inédite pour l'humanité peut fragiliser les identités individuelles, mais aussi susciter des heurts entre des groupes culturels. (p. 5)

Autant dire que les difficultés du vivre-ensemble actuel sont une réalité que la littérature ne pouvait en aucun cas esquiver pour donner à comprendre dans son ensemble le vécu migratoire, du point de vue tant de l'immigrant que de l'habitant du pays dit « d'accueil ». Dans Le Jeu des sept cailloux, le rejet s'écrit à la troisième personne dans la perspective de Larissa: «Les regards froids de certains passants blessent Larissa autant que des fusils.» Cette comparaison d'égalité entre les blessures en provenance des fusils des snipers et les atteintes venant des passants de Rouen parvient à donner idée de la souffrance endurée par l'immigrée, en proie à la désapprobation, au reproche et au rejet de sa vie, de son être.

Dans La fille qui parle à la mer, le vivre-chacun chez soi s'exprime cette fois au discours direct, adressé à Oyana : «Des gens comme toi, il y en a plein. Il en arrive tous les jours. Comment on fait pour nourrir tout le monde? Et puis il paraît que les autres sont tout près, qu'ils veulent prendre la ville 
aussi. Ils. Ils sont là. Ils arrivent. Les autres. » (p. 15). Dans ce passage, Oyana n'est pas appréhendée par le locuteur comme une petite fille singulière et entière mais comme une petite partie d'un tout, informe, énorme, reproductible, une masse de migrants menaçants. La répétition du « ils », d'abord isolés puis suivis de verbes de présence et de mouvement, encore redoublés par leur désignation sous le terme "les autres", marque la distance et la frontière qu'opère le locuteur entre lui, les habitants de son village, et l'altérité, celui qui vient, pour lui manger le pain dans la main.

$\mathrm{Si}$, dans cet album, la peur de ne plus avoir de quoi se nourrir peut apparaître légitime et en tous cas, être comprise, il en va tout autrement dans l'album des Vitalabri, dont les raisons exposées de rejet sont moquées par le narrateur :

Là, il faut vous dire que certaines familles vitalabraises ont beaucoup d'enfants, beaucoup trop, d'autres par contre n'en ont pas du tout, ce qui est trop peu, et c'est aussi pourquoi on n'aime pas les Vitalabri. Comment? Vous n'avez pas compris, là, le pourquoi? C'est simple, parce qu'ils ont trop d'enfants ou pas assez. C'est clair? Voilà! Ah, j'avais oublié de vous dire, on n'aime pas les Vitalabri aussi à cause de la couleur de leur peau, trop blanche, ou trop marron, ou trop jaune, ou trop rouge, d'ailleurs on a déjà zigouillé un grand nombre de ceux qui avaient la peau rouge, qui vivaient tout nus et qui faisaient peur aux enfants des autres couleurs de peau, les visages pâles. (p.11)

Ainsi, que ce soit par le biais de la perspective du personnage migrant ou de celle du personnage se sentant menacé par l'immigrant, ou encore de celle du narrateur qui tourne en dérision les motifs dérisoires, multiples et contradictoires d'exclusion, l'enfant-lecteur ne pourra manquer de comprendre que le parcours des difficultés de l'émigré ne 
s'arrête pas lorsqu'il se mue en immigré et que l'accueil se combine bien souvent avec des formes sournoises et mesquines de rejet.

Puisqu'il s'agit d'un corpus de six albums, nous ne saurions nous contenter d'une analyse du texte sans mentionner l'importance du rôle des images dans la compréhension du phénomène de migration. Cette importance est évidemment inégale, moindre dans La fille qui parle à la mer / Le garçon au chien parlant, qui ne comporte qu'une double page illustrée au centre de l'album, en plus de la couverture. Forte dans les albums "Français d'ailleurs », Le Jeu des sept cailloux et Les Vitalabri, dès lors que les images accompagnent régulièrement et fidèlement le texte. Primordiale dans Akim court, au point de renverser la tendance et de donner l'impression d'un texte accompagnant les images. L'auteurillustratrice belge Claude K. Dubois a réalisé environ 80 albums jeunesse et exprimé le rôle que jouent les illustrations en lien avec les personnages :

L'émotion est la seule force directrice dans mon travail. Je ne dessine pas pour le côté graphique, celui-ci me sert en fait à traduire avec force les sentiments de mes personnages : la joie, la tristesse et, toujours en filigrane, la fragilité et l'étonnement face au monde qui nous entoure. Le regard des enfants croisera celui de mes personnages. Pour que cette rencontre magique ait lieu, il faut que ce regard soit vrai, sincère et vivant. (page web)

Le crayonné de l'auteur illustratrice est effectivement remarquable, empreint d'une grande simplicité, d'une économie dans les teintes (le gris du crayon de bois accompagné parfois de légères teintes orangées), d'un 
minimalisme de trait, d'un flou du représenté, enfin, d'une candeur et d'une innocence qui savent toucher notre humanité.

De plus, les images, occupant la plus grande part de l'album, ne se contentent pas d'apporter la confirmation de ce que dit le texte mais le dépassent, vont au-delà. Lorsque Akim, tout petit garçon menacé par la guerre, fuit son pays, on comprend, en suivant le fil des images, qu'il a pensé, dans la pire des tourmentes, à emmener avec lui son nounours. Sur la page de gauche, est représenté un bras tendu vers la petite peluche, et sur celle de droite, le petit garçon est agenouillé au sol, tandis qu'il adresse un câlin au doudou terrorisé. Il est touchant de constater qu'un aussi petit garçon, en butte à la guerre et à la destruction, et qui aurait donc tant besoin de protection, sache pourtant puiser en lui des forces d'altruisme et d'affection. Or, cela, les mots ne le disent pas, seules les images racontent cet épisode marquant de l'histoire. Ailleurs dans l'album, les images sont habiles à signifier le contraste entre oppression et désir de liberté, innocence et armes menaçantes, les mains qui empoignent et les mains qui se tendent. Pour accentuer cette dichotomie, l'usage de la métonymie est fréquent; par exemple, les armes pour les soldats. On note aussi, en s'arrêtant sur quelques autres illustrations, la vitesse, le déchaînement et le chahut de la guerre, en opposition avec le silence, la dignité et l'immobilité de l'enfant. Ainsi, les illustrations des albums jeunesse de la migration peuvent se faire discrètes ou suivre avec régularité la narration et ses petits faits, ou de façon plus ambitieuse encore, participer d'une compréhension empathique d'un personnage et, par extension, de la situation, réelle et dramatique, de tous les réfugiés. 
Cette situation est toujours celle de victimes mais les albums se refusent unanimement au pathétique. Nous avons vu plus haut que le pendant du statut de victime peut-être l'héroïsation de personnages forts et courageux, mais l'immigré apparaît aussi comme une opportunité d'ouverture sur l'altérité et d'échange interculturel. Ce passage entre différentes cultures est au cœur-même du projet de la collection «Français d'ailleurs », justifiant ainsi sa raison d'être :

Pourquoi? L'histoire de l'immigration aux XIX et XX ${ }^{\mathrm{e}}$ siècles a façonné le paysage social et culturel de la France contemporaine. Raconter les destins singuliers des immigrants en France permet de redécouvrir l'histoire de notre pays et de créer un dialogue interculturel, loin de la simplification, de la schématisation et des clichés. (avant-propos)

Dans Les deux vies de Ning, le lecteur aura ainsi l'occasion de découvrir les traditions du Nouvel An chinois en Chine, différentes de celles des Chinois résidant en France (p. 43). Il pourra aussi être attentif à l'exposé sur la Chine que la classe de sixième a préparé et présenté en classe de français langue étrangère; c'est à cette occasion, en effet, que le texte se donne pour mission d'éloigner les clichés :

Les sixièmes sont venus avec leurs notes... et toutes leurs idées fausses. Je les ai écoutés, médusé, dire que les Chinois :

mangent du riz à tous les repas, gluant, en pâtes, gâteaux, glaces, brioches;

font du judo, du taekwondo ou du karaté;

lisent surtout des mangas;

font travailler des enfants dans des usines. (p. 57)

Ning va commencer par corriger les idées fausses et, dans un même élan, complétera l'image de la Chine en sélectionnant ses aspects les plus remarquables. L'anaphore traduit son emballement : «Je leur ai dit [...] J'ai dit [...] J'ai dit [...]. » Ces 
échanges ne sont pas seulement une opportunité d'apprentissage de la culture chinoise pour la classe de français mais aussi pour Ning qui est amené, pour la première fois, à regarder son pays de l'extérieur, ce qui, à bien des égards, notamment identitaire, sera révélateur :

Je sentais mes lèvres trembler, mon souffle court, je parlais vite, je défendais la Chine comme une personne que j'aime et à qui on fait mal. Comme j'aurais défendu ma mère. Je me suis rendu compte à quel point la Chine me manquait. À quel point je l'aimais. Comme on s'aperçoit mieux qu'on aime, dans l'absence. (p. 59)

Ning prend conscience de son identité de Chinois quand il exprime ses sentiments pour la Chine mais aussi quand il porte un regard sur des coutumes françaises différentes des siennes. Lorsque sa maman lui annonce qu'elle a trouvé un vrai travail, il est très fier et se dit en lui-même, mais à l'adresse de sa mère : "J'ai presque envie de t'embrasser, comme font les enfants français à la sortie de l'école à côté du collège, des baisers sur la joue avec les bras autour du cou, on dirait qu'ils veulent s'enfoncer dans le corps de leur mère, j'avais jamais vu ça avant.»(p.65) Ainsi, la présence du jeune Ning en France constitue un bénéfice partagé d'interculturalité dans quatre sens, car les échanges permettent de porter un regard neuf sur le pays étranger aussi bien que sur le sien, pour le résidentsouche comme pour le résident nouvellement arrivé.

Ce passage des cultures est aussi favorisé, dans la collection "Français d'ailleurs", par le lexique de la langue étrangère intégré tel quel dans les textes en français. L'album Joao ou l'année des révolutions compte onze mots portugais parsemés ici ou là dans le texte. Ce lexique porte sur les spécialités gastronomiques, par exemple le «bacalhau : morue. 
Les plats utilisant la morue, très variés, sont une des grandes spécialités de la cuisine portugaise. » (p. 79), dit-on, ou encore sur la musique, « braguesa : viola braguesa, guitare de la région de la ville de Braga » (ibid.). Bon nombre de mots se réfèrent à la situation politique, comme la «PIDE : Police de la dictature salazariste » et quelques-uns à la religion, avec notamment la mention de la ville de Fatima, l'un des plus importants lieux de pèlerinage en Europe. L'intégration de ce lexique exotise un tant soit peu les textes et conduit le lecteur à la recherche et à la découverte de nouveaux mots et donc d'une nouvelle culture, ce qui accroît non seulement sa compréhension de la migration mais affûte aussi son envie de découvrir l'autre, son altérité, sa différence. Les camarades de Joao pourront apprendre à jouer à la malha, "jeu traditionnel villageois », de même que, dans Le Jeu des sept cailloux, les enfants pourront apprendre à jouer à ce jeu tchétchène ou encore à préparer le tchépalgash, spécialité culinaire. Ces activités sont aussi susceptibles d'intéresser l'enfant-lecteur et donc de l'amener vers une compréhension de la potentielle richesse d'une ouverture à l'altérité. Ainsi, l'immigré-personnage n'est pas confiné à un statut de victime mais présenté comme vecteur d'opportunités. Encore faut-il le comprendre et choisir de le saisir. En effet, dans l'album des Vitalabri, l'aîné parcourt différents pays, chez les schnellbunker (pays germanique), chez les prestocasa (pays du sud), et il subit rejet, humiliation, expulsion, jusqu'à son arrivée chez les quickhome (on pense aux États-Unis), où il est véritablement accueilli et passe ainsi de petit vitalabri à grand concertiste.

L'album des Vitalabri tranche avec les autres productions du corpus sélectionné du fait qu'il traite le sérieux du sujet de la migration sur un ton léger, humoristique et décalé. À notre avis, l'humour de Jean-Claude Grumberg participe aussi, par une 
dédramatisation-poétisation ludique, à la compréhension des tenants et des aboutissants des mouvements de population. D’emblée, le texte pose aux enfants une question : "Connaissezvous les Vitalabri? », contraction de « vite à l'abri » et peut-être aussi de «vital abri ». Le vitalabri est défini comme celui qui est partout et nulle part chez lui, mais surtout nulle part. Sa caractéristique principale est de n'être pas aimé par ceux qui sont nés quelque part et qui ne sont chez eux que là où ils sont nés. Pourquoi n'aime-t-on pas les Vitalabri? S'ensuit une liste de raisons absurdes et contradictoires, à l'instar de celle-ci : « Certains disent - surtout ceux qui ont le nez rond - qu'ils n'aiment pas les Vitalabri parce que ceux-ci ont le nez pointu, et ceux qui ont le nez pointu, eux, n'aiment pas les Vitalabri parce qu'ils trouvent leur nez trop rond. » (p. 9) Le comique provient de l'accumulation de ces raisons farfelues qui se jouent de toute logique jusqu'à s'annuler les unes les autres. L'enfant sera conduit, par le biais de l'humour, à conclure que les Vitalabri sont ses semblables et qu'aucune raison sérieuse ne justifie leur rejet. Les indications spatiales sont également en proie à des allers retours fulgurants, grâce au procédé d'épanorthose, lequel consiste, pour le narrateur, à apporter des corrections à ce qu'il vient de dire, conférant ainsi légèreté et spontanéité aux propos relatés : «La première nuit qu'ils passèrent loin de chez eux - ou plutôt non, pas très loin de leur point de départ, mais très loin en revanche de leur point d'arrivée, étant donné qu'ils ne connaissaient pas ce point, et qu'ils avaient déjà oublié le point d'où ils étaient partis le matin-même.» (p.19) On note aussi beaucoup d'apostrophes au lecteur, "vous savez bien » (p. 19), d'interjections, « et hop» (p. 24), de pseudo-questions «Pour manger? Comment faisaient-ils pour manger? C'est une très bonne question, je vous remercie de me l'avoir posée [...]. » 
(p. 22) qui favorisent la captation de l'attention en accentuant l'impression de déroulé de l'histoire dans l'immédiateté. C'est toujours avec charme, légèreté et humour que les pratiques des bourreaux sont dénoncées. Ainsi des mensonges du passeur qui cherche à séduire la toute petite Vitalabri :

- Moi, je veux bien manger des noix, murmura la plus petite.

- De l'autre côté, tu en mangeras, y en a plein les trottoirs.

- Que font les noix sur les trottoirs?

- Elles y poussent afin que les petits vitalabrais puissent les cueillir et les manger sans se fatiguer. (p. 26)

L'aspect charmant et humoristique provient aussi de la musicalité de certains passages dialogués et rythmés. On y reconnaît d'ailleurs le Jean-Claude Grumberg dramaturgescénariste, écrivain pour enfants. À la famille Vitalabri qui n'a pas de quoi le payer, le passeur s'écrie ainsi :

- Donnez-moi vos instruments de musique et surtout le violon du garçon.

- Il n'en est pas question! hurla l'aîné, en serrant son violon. Mes livres, après mon violon, non non non!

- Non non non! crièrent en écho le reste des enfants.

- Bon bon bon, alors votre édredon et un ou deux pantalons. (p. 30)

La répétition des «non non non » à laquelle répond le «bon bon bon», en écho aux rimes de "garçon, violon, question, édredon et pantalons », est telle une dramatisation, au sens théâtral du terme, qui vient paradoxalement, au moyen de la musique et de l'humour, dédramatiser la situation, ce qui n'empêchera pas l'enfant-lecteur de comprendre, tout en riant, que le passeur, avide et menteur, nourrit de bien mauvaises intentions. Il nous semble que conter l'immigration, ses bonheurs, ses laideurs, le tout sur un mode ludique, léger, poétique et optimiste sert à la fois le plaisir et la compréhension 
de l'enfant d'un phénomène que Les Vitalabri rendent universel, drôle et touchant.

Au terme de cette étude portant sur un corpus éclectique de six albums de littérature jeunesse de l'immigration, il apparaît que chacune de ces histoires, dans des modalités majoritairement communes et parfois singulières, contribue effectivement à la compréhension du monde en mouvement dans lequel vivent les enfants. Des ouvrages à visée purement informative comme Vivons ensemble, Pour répondre aux questions des enfants sur l'immigration, ou encore L'Immigration à petits pas seront certes plus prolixes, plus complets et plus structurés dans l'accès aux informations visant la compréhension mais les albums en constituent toutefois, à notre avis, le pendant nécessaire. En effet, l'enfant reste à distance de l'ouvrage d'informations alors qu'il expérimente les aventures migratoires dans des albums dont les personnages, souvent des enfants, favorisent le procédé d'identification-projection. De même, le livre documentaire renseigne sur la grande histoire tandis que la littérature donne à vivre la petite en la contextualisant et en l'exemplifiant dans la grande. Ainsi, le premier s'efforce de maintenir neutralité et distance tandis que le second joue de la visée testimoniale pour susciter l'empathie, avec force poésie, procédés ludiques, contrastes et images, tant stylistiques que graphiques.

En explorant l'ensemble de ces albums et en rapportant leur contenu aux problématiques de l'immigration telles que décrites dans les ouvrages documentaires, on remarque que la 
grande majorité d'entre elles s'y retrouvent, si bien que la littérature apparaît apte, dans l'économie de ses petits formats jeunesse, à dire dans toute son extension - ou presque - les réalités de l'immigration. Toutefois, si l'on s'arrête, accessoirement, à remarquer l'absence de certaines thématiques préoccupantes à l'instar de celle du climat, laquelle se profilera certainement dans les fictions de migrations jeunesse à venir, c'est la représentation laudative du migrant qui retient dans l'immédiat toute notre attention. Bénéfique, parce que constitutive d'un contrepoids à la vision médiatique de l'immigrant à problème. Pourtant, il n'en demeure pas moins que tous les immigrés ne sont pas sympathiques, ni héroïques, et certains d'entre eux posent même un véritable défi au vivreensemble. Or, aucune de ces fictions ne problématise vraiment les incompatibilités religieuses, culturelles et politiques. Seul l'album des Vitalabri a le mérite de préciser qu'il existe des Vitalabri polis et d'autres, très impolis, soit par manque d'éducation, soit à cause des douloureuses épreuves qu'ils ont eu à endurer. Les gentils Vitalabri ne sont donc pas exemplaires de tous les Vitalabri.

Si l'on s'accorde à considérer la question du vivreensemble comme fondamentale à la compréhension du monde d'aujourd'hui, on pointerait alors les failles de ces fictions dans l'absence de problématisation de cette notion. Les contraintes éditoriales, plus fortes encore dans la littérature destinée à la jeunesse, expliqueraient peut être pourquoi tensions et nécessités de compromis entre peuples, gouvernements et religions, ne se trouvent pas reflétées dans des vies ouvertes mais parfois aussi soumises à l'altérité et à sa différence. 
Nous espérons, pour l'avenir, des albums qui embrasseront pleinement cette notion, «car dans l'immigration, c'est [certes] l'homme, la femme, les enfants qui sont en jeu. Une part d'humanité commune, universelle, [mais aussi une autre, nous l'ajoutons, à la fois culturelle, sociale, singulière et différentielle] qui peut et doit être évoquée [mais aussi problématisée] et dite à tout âge, comme une [véritable] compréhension du monde dans lequel on vit » (Falaise, p. 261).

\section{Bibliographie}

BAnon, Patrick et Anne-Lise Boutin. (2016), Guide du mieuxvivre ensemble. Ma laïcité, ma religion, mon identité, Paris, Actes Sud Junior.

DuboIs, Claude K. (2012), Akim court, Paris, L'école des loisirs, coll. « Pastel » (non paginé).

-. L'école des loisirs, <http://www.ecoledesloisirs.fr/phpedl/auteurs/fiche-auteur-nvo.php? codeauteur=78>.

FALAISE, Benoît. (2012), " Postface », dans Mustapha Harzoune et Samia Messaoudi, Vivons ensemble. Pour répondre aux questions des enfants sur l'immigration, Paris, Albin Michel Jeunesse.

GALEA, Claudine. (2013), La fille qui parle à la mer / Le garçon au chien parlant, Arles, Éditions du Rouergue, coll. «Boomerang ».

Goby, Valentine. (2013), Les deux vies de Ning, Paris, Éditions Autrement Jeunesse, coll. «Français d'ailleurs ».

-. (2010), João ou l'année des révolutions. Paris, Éditions Autrement Jeunesse, coll. «Français d'ailleurs ». 
GrumberG, Jean-Claude. (2014), Les Vitalabri, Paris, Actes Sud Junior.

HARzoune, Mustapha et Samia MESSAOUdi. (2012), Vivons ensemble. Pour répondre aux questions des enfants sur l'immigration, Paris, Albin Michel Jeunesse.

HÉRAN, François. (2007), « Le temps des immigrés. Essai sur le destin de la population française », La République des Idées, <http://www.repid.com/Le-temps-des-immigres.html>.

LAMOUREUX, Sophie et Guillaume LONG. (2011), L'Immigration à petits pas, Paris, Actes Sud Junior.

MARTINACHE, Igor. (2007), "François Héran, Le Temps des immigrés. Essai sur le destin de la population française», Lectures, <https://lectures.revues.org/434>.

SAMPIERO, Dominique. (2010), Le Jeu des sept cailloux, Paris, Éditions Grasset-Jeunesse, coll. «Lampe de poche» (non paginé).

SCHNEIDER, Anne. (2013), La Littérature de jeunesse migrante. Récits d'immigration de l'Algérie à la France, Paris, L'Harmattan, coll. « Espaces littéraires ».

\section{Résumé}

«L'immigration [...] doit être [...] dite à tout âge, comme une compréhension du monde dans lequel on vit.» Partant du constat émis par Benoît Falaise, nous sondons six ouvrages de littérature jeunesse dont la thématique est l'immigration. Nous postulons que ces albums visent une compréhension empathique dans un dialogue entre désir d'informer et d'émouvoir, au moyen de procédés esthétiques variés, tant visuels qu'écrits. L'analyse, centrée sur les textes, recourt aussi 
à des ouvrages documentaires pour examiner les entrées thématiques textualisées, particulièrement celle du «vivre ensemble », au cœur des grands débats de notre société.

\begin{abstract}
"Immigration [...] must be [...] told at any age, as an understanding of the world in which we live." On the basis of that point made by Benoît Falaise, we work on six stories for youth whose topic is immigration. We assume that these albums aim to gain empathetic understanding in a dialogue between a desire to inform and to move children, through visual and written aesthetic process. The analysis is focused on the texts but also captures the themes addressed in nonfiction books, especially that of "living together ", in the heart of major societal debates.
\end{abstract}

